\title{
PRODUCTS OF THREE FACTORIALS
}

\author{
A. DUJELLA, F. NAJMAN, N. SARADHA AND T. N. SHOREY
}

\begin{abstract}
We study a question of Erdős and Graham on products of three factorials being a square.
\end{abstract}

\section{INTRODUCTION}

For any integer $n>1$ denote by $P(n)$ and $Q(n)$ the greatest prime factor and the square free part of $n$ and put $P(1)=Q(1)=1$. For any prime $p$, let $\operatorname{ord}_{p}(n)$ denote the largest power of $p$ dividing $n$. For any set $A$ of positive integers let

$$
m(A)=\prod_{a \in A} a !
$$

and $M(A)$ denote the largest integer in $A$. In 1975, Erdős and Selfridge [7] proved a remarkable result that a product of two or more consecutive positive integers is never a square or a higher power. Continuing this line of investigation, Erdős and Graham [9] asked if a product of two or more disjoint blocks of consecutive integers can be a square or a higher power. It was noted by Ulas [15] that if all the blocks are of length exactly 4 and if the number of such blocks is large enough, then the product takes on square values infinitely often. In subsequent papers Bauer and Bennett [1] and Bennett and Luijk [2] gave further possibilities when such a product is a square infinitely often. In this paper, we consider a product of factorials being a square i.e., we search for positive rational integral solutions to the Diophantine equation

$$
\prod_{k=1}^{t} a_{k} !=y^{2}
$$

with $a_{1} \geq a_{2} \geq \cdots \geq a_{t}>1$ and $y>1$. By canceling out an even number of equal factorials on the left hand side, we may assume that

$$
a_{1}>a_{2}>\cdots>a_{t}>1 \text {. }
$$

2000 Mathematics Subject Classification. Primary: 11D61; Secondary: 11Y50.

Key words and phrases. Factorials, square free factor. 
In [8], the above equation was studied when $a_{1}$ is given and $t$ is minimal such that (1) has a solution. It was shown in [8] that the set of possible values of the left hand side of (1) is sparse. Hence it may very well be that (1) has no or very few solutions. Define $F_{0}=\emptyset$ and for $k \geq 1$, let

$$
\begin{aligned}
F_{k}= & \{n: \text { there exists some set } A \text { with }|A| \leq k, \\
& M(A)=n \text { and } m(A) \text { is a square }\}
\end{aligned}
$$

and

$$
D_{k}=F_{k} \backslash F_{k-1} \text {. }
$$

Suppose the equation has a solution $a_{1}, \cdots, a_{t}$ then $a_{1} \in F_{t}$. Thus in order to study the equation (1) Erdös and Graham investigated the sets $F_{k}$ and $D_{k}$.

1.1. Properties of the sets $F_{k}$ and $D_{k}$. (i) No prime belongs to any $F_{k}$.

(ii) $D_{1}=F_{1}=\{1\}$ since $n$ ! for $n \geq 2$ is never a square.

(iii) Let $A=\left\{a^{2}, a^{2}-1\right\}$ with $a>1$. Then $t=2, a_{1}=a^{2}, a_{2}=a_{1}-1$ and $m(A)$ is a square. Hence $a^{2} \in F_{2}$. By [7], it follows that $D_{2}=$ $\left\{a^{2}, a>1\right\}$.

(iv) Let $a>1, b>1$ and $A=\left\{a^{2} Q(b !), a^{2} Q(b !)-1, b\right\}$. Then $t=$ $3, a_{1}=a^{2} Q(b !), a_{2}=a_{1}-1, a_{3}=b$ and $m(A)$ is a square. Hence $a^{2} Q(b !) \in D_{3}$.

(v) Let $x, y$ be solutions of the Pell's equation

$$
u x^{2}-v y^{2}=1
$$

where $u v=Q(a !)$ for some $a>1$ and $\operatorname{gcd}(u, v)=1$. Let $A=$ $\left\{u x^{2}, v y^{2}-1, a\right\}$. Then $t=3, a_{1}=u x^{2}, a_{2}=a_{1}-2, a_{3}=a$ and $m(A)$ is a square. Hence $u x^{2} \in D_{3}$ when $u$ is not a square. This example is slightly different from the one given in [7] where $u v$ is taken to be equal to $a$ !.

Erdös and Graham predict that there are perhaps only finitely many elements of $D_{3}$ apart from the two classes of integers given in (iv) and (v). This problem is still open. On the other hand, they showed that $D_{3}$ is sparse by proving

$$
D_{3}(X)=o(X)
$$

where $D_{3}(X)$ denotes the number of elements of $D_{3}$ which do not exceed $X$. The above result was improved by Saradha and Shorey [14] to

$$
D_{3}(X)=O\left(\frac{X \log _{3} X}{\log X \log _{2} X}\right) .
$$


Their work depends on an application of Jutila's result on exponential sums and known estimates for the Dickman's function which counts integers with small prime factors.

1.2. About $D_{3}$. In the two classes of examples in (iv) and (v) we have $a_{2} \geq a_{1}-2$. There are examples where $a_{2}=a_{1}-3$. For instance,

$$
10 ! 7 ! 6 ! ; 50 ! 47 ! 3 ! ; 50 ! 47 ! 4 !
$$

are all squares. Erdős and Graham asked if there are other examples. i.e., does the equation

$$
a_{1} !\left(a_{1}-3\right) ! a_{3} !=y^{2}
$$

have any other solution apart from the three examples given above? We prove

Theorem 1.1. Let $a_{3} \leq 100$. Then all the solutions of (3) are given by

$\left(a_{1}, a_{3}\right) \in\{(10,6),(50,3),(50,4),(324,26),(352,13),(442,18),(2738,26)\}$.

Note that (3) can be re-written as

$$
a_{1}\left(a_{1}-1\right)\left(a_{1}-2\right) a_{3} !=y^{2}
$$

The left hand side of (4) is a product of two non-overlapping blocks, one of length 3 and the length of the other is not fixed. These cases are not covered in [1] and [2]. For $x \in \mathbb{R}$, by $\lceil x\rceil$ we denote the ceiling function of $x$, i.e. the smallest integer not less than $x$. We show

Theorem 1.2. Let $h=\pi\left(a_{3}\right)-\pi\left(a_{3} / 2\right)$. Then

(a) $a_{1}>\left(a_{3} / 2\right)^{\lceil h / 3\rceil}$.

(b) No term $N \in\left\{a_{1}, a_{1}-1, a_{1}-2\right\}$ is of the form $\alpha N_{1}$ where $N_{1}=P_{1}^{\theta_{1}} \cdots P_{s}^{\theta_{s}}>1$ with $P_{i}$ 's primes, $\theta_{i}$ odd, $\Omega\left(N_{1}\right) \leq\lceil h / 3\rceil$ and $\alpha \leq \frac{a_{3}^{\lceil h / 3\rceil-\Omega\left(N_{1}\right)}}{2^{\lceil k / 3\rceil}}-\frac{2}{a_{3}^{\Omega\left(N_{1}\right)}}$, where $\Omega\left(N_{1}\right)$ denotes the number of prime factors of $N_{1}$ counted with multiplicity.

(c) No term $N$ is of the form $\alpha N_{1}$ where $N_{1}=P_{1}, P_{1}^{3}, P_{1} P_{2}, P_{1} P_{2} P_{3}$ and $\alpha \leq 64393,6,637,6$, respectively, except when $\left(a_{1}, a_{3}\right) \in\{(10,6),(50,3),(50,4),(324,26),(352,13),(442,18),(2738,26)\}$.

\section{Proof of Theorem 1.1}

Equation (3) can be re-written as

$$
a_{1}\left(a_{1}-1\right)\left(a_{1}-2\right)=Q\left(a_{3} !\right) Y^{2} .
$$


Putting $a_{1}-1=X^{\prime}$ and multiplying by $\left(Q\left(a_{3} !\right)\right)^{3}$ the equation is transformed to the elliptic equation

$$
X\left(X^{2}-b^{2}\right)=Y^{2}
$$

where $X=Q\left(a_{3} !\right) X^{\prime}, Y=\left(Q\left(a_{3} !\right)\right)^{2} Y^{\prime}$ and $b=Q\left(a_{3} !\right)$. Actually, (6) is the so called congruent number elliptic curve. For $a_{3} \leq 53, a_{3} \notin$ $\{32,38,40,47,51\}$, we were able to find all integral points $(X, Y)$ on the elliptic curve (6) by using the function IntegralPoints in Magma [3]. However, we are interested only in those integer points for which $X$ is divisible by $b$. The integer points satisfying this additional condition give exactly the solutions given in Theorem 1.1.

For the remaining values of $a_{3}$ we were not able to find all integral points on (6), mainly because we were unable to find the generators of the Mordell-Weil group of the curve (6). Therefore, in these cases we take a different approach by transforming our problem into a family of systems of Pellian equations. Since, $\operatorname{gcd}\left(a_{1}, a_{1}-1\right)=\operatorname{gcd}\left(a_{1}-1, a_{2}-\right.$ $1)=1$ and $\operatorname{gcd}\left(a_{1}, a_{1}-2\right) \in\{1,2\}$, from (5) we get:

$$
a_{1}=b_{1} x^{2}, \quad a_{1}-1=b_{2} y^{2}, \quad a_{1}-2=b_{3} z^{2}
$$

or

$$
a_{1}=2 b_{1} x^{2}, \quad a_{1}-1=b_{2} y^{2}, \quad a_{1}-2=2 b_{3} z^{2},
$$

where $b_{1} b_{2} b_{3}=b$. These relations induce the system of Pellian equations

$$
b_{1} x^{2}-b_{2} y^{2}=1, \quad b_{1} x^{2}-b_{3} z^{2}=2, \quad b_{2} y^{2}-b_{3} z^{2}=1,
$$

and

$$
2 b_{1} x^{2}-b_{2} y^{2}=1, \quad b_{1} x^{2}-b_{3} z^{2}=1, \quad b_{2} y^{2}-2 b_{3} z^{2}=1,
$$

respectively.

The number of such systems can be very large $\left(3^{\omega(b)}\right.$, where $\omega(b)$ denotes the number of prime factors of $b$ ). However, we expect that most of them have no solutions. We will follow an approach from $[5,6]$. So we start by eliminating those systems which are not locally solvable. Indeed, almost all system can be shown to be non-solvable by considering solvability modulo 8 and modulo $p$ for primes $p$ dividing $b$ (the solvability conditions can be written in terms of Legendre symbols, e.g. for the first system we have $\left(\frac{-b_{2}}{p_{1}}\right)=1$ and $\left(\frac{-2 b_{3}}{p_{1}}\right)=1$ for all primes $p_{1}$ dividing $b_{1}$, and similar conditions for primes dividing $b_{2}$ and $\left.b_{3}\right)$. For each value of $a_{3}$ in the considered range, there remains only a few locally solvable systems. Note that we always have the system

$$
2 x^{2}-y^{2}=1, \quad x^{2}-b_{3} z^{2}=1, \quad y^{2}-2 b_{3} z^{2}=1,
$$


in which each particular equation is certainly solvable in positive integers. However, from the system ( 7 ) we get $y^{2}+1=2 x^{2}, y^{2}-1=2 b_{3} z^{2}$ and hence

$$
y^{4}-b_{3}(2 x z)^{2}=1 \text {. }
$$

Now we can apply a result due to Cohn [4] which says that the only possible solutions of the equation $X^{4}-D Y^{2}=1$ in positive integers are given by $X^{2}=u_{0}$ and $X^{2}=2 u_{0}^{2}-1$, where $u_{0}+v_{0} \sqrt{D}$ is the fundamental solution of Pell's equation $u^{2}-D v^{2}=1$. For $a_{3} \leq 88$, we were able to compute the fundamental solution by using the function quadunit in Pari [12]. For $89 \leq a_{3} \leq 100$, the fundamental units become too large to be computed by standard methods, as they grow exponentially in the size of the discriminant of the quadratic field. Because of this, we used compact representations of fundamental units and the algorithm for modular arithmetic on such representations described in [11].

We find that for $a_{3} \leq 100$ the equation (8) has a solution in positive integers only for $a_{3}=3,4,18$, and these cases were already handled by elliptic curve approach.

The remaining Pellian equations, not eliminated by the previously explained methods, have the shape $\alpha x^{2}-\beta y^{2}=1$ or 2 , where $\alpha>1$, $\beta>0$. The criteria for solvability of such equations was given by Grelak and Grytczuk [10] in terms of the fundamental solution $\left(u_{0}, v_{0}\right)$ of the equation $u^{2}-\alpha \beta v^{2}=1$. For the equation $\alpha x^{2}-\beta y^{2}=1$, the criterion is that $2 \alpha \mid\left(u_{0}+1\right)$ and $2 \beta \mid\left(u_{0}-1\right)$, while for $\alpha x^{2}-\beta y^{2}=2$, $\alpha \mid\left(u_{0}+1\right)$ and $\beta \mid\left(u_{0}-1\right)$.

After an application of these criteria, only three systems remain unsolved. We list them explicitly. Each of them can be solved by using some divisibility properties of the corresponding fundamental units.

For $a_{3}=32$, we have to consider the system

$$
x^{2}-1964315 y^{2}=1, \quad x^{2}-34 z^{2}=2 .
$$

The second equation clearly implies that $3 \mid x$. Since the fundamental solution $\left(x_{0}, y_{0}\right)$ of the first equation has the property $3 \mid y_{0}$, we conclude that also $3 \mid y$. But it is clear that there is no solution of the first equation such that both $x$ and $y$ are divisible by 3 .

For $a_{3}=54$, the remaining system is

$$
63017 x^{2}-y^{2}=1, \quad 63017 x^{2}-39407479 z^{2}=2 .
$$

From the fundamental solution of the first equation we get that $37 \mid x$. But this contradicts the second equation since also 37|39407479.

For $a_{3}=84$, the remaining system is

$$
163373405489 x^{2}-17755 y^{2}=1, \quad 17755 y^{2}-16077666 z^{2}=1 .
$$


Now we get contradiction since $7 \mid 163373405489$, while from the fundamental solution of the second equation it follows that also $7 \mid y$.

\section{Proof of Theorem 1.2}

We make the following observations:

Let $S=\left\{a_{1}, a_{1}-1, a_{1}-2\right\}$.

1) None of the elements of $S$ is a prime since $a_{1}-3=a_{2}>a_{3}$.

2) The gcd of any two of the elements of $S$ is either 1 or 2 .

3) $a_{3} \geq 101$ by Theorem 1.1. Then $k \geq 11$. This can be checked for $a_{3} \geq 250$ by estimates for $\pi(X)$ (see [13]) and for $101 \leq a_{3}<250$ by the exact values of $\pi(X)$.

Proof of (a).

All the primes dividing $a_{3}$ ! to an odd power must divide the terms in $S$. This is true in particular for the primes in the range $\left(a_{3} / 2, a_{3}\right]$. Since there are $k$ primes in $\left(a_{3} / 2, a_{3}\right]$, there is a term $N \in S$ which is divisible by at least $\lceil h / 3\rceil$ primes. Thus

$$
a_{1} \geq N>\left(a_{3} / 2\right)^{\lceil h / 3\rceil} .
$$

Proof of (b).

Suppose a term $N \in S$ satisfies the given conditions of (b). By (4), each of the primes occurring in $N$ is $\leq a_{3}$, so we get

$$
a_{1} \leq N+2 \leq\left(a_{3} / 2\right)^{\lceil h / 3\rceil} .
$$

This is a contradiction to (9), proving (b).

Proof of (c).

By Theorem 1.1 we may assume that $a_{3} \geq 101$. Then $h \geq 11$. It follows by (9) that $a_{1}>a_{3}^{4} / 16$. By (b), there is no term $N \in S$ of the form $\alpha N_{1}$ with $N_{1} \in S_{1}=\left\{P_{1}, P_{1}^{3}, P_{1} P_{2}, P_{1} P_{2} P_{3}\right\}$ and $\alpha \leq \frac{a_{3}^{4-\Omega\left(N_{1}\right)}}{16}-$ $\frac{2}{a_{3}^{\Omega\left(N_{1}\right)}}$. Since $a_{3} \geq 101$, it follows that there is no term $N=\alpha N_{1}$ with $N_{1} \in S_{1}$ and $\alpha \leq \frac{101^{4-\Omega\left(N_{1}\right)}}{16}-\frac{2}{101^{\Omega\left(N_{1}\right)}}$. Thus we get $\alpha \leq 64393,6,637,6$ according as $N_{1}=P_{1}, P_{1}^{3}, P_{1} P_{2}, P_{1} P_{2} P_{3}$, respectively. This proves (c).

\section{REFERENCES}

[1] M. BAuer AND M. BennetT, On a question of Erdös and Graham, L'enseignement Math. 53 (2008), 259-264.

[2] M. Bennett AND R. VAn LuiJk, Squares from blocks of consecutive integers: A problem of Erdös and Graham, Indag. Math. (N.S.) 23 (2012), 123-127.

[3] W. Bosma, J. J. Cannon, C. Fieker And A. Steel (eds.), Handbook of Magma functions, Edition 2.18 (2011).

[4] J. H. E. Cohn, The Diophantine equation $x^{4}-D y^{2}=1$, II, Acta Arith. 78 (1997), 401-403. 
[5] A. Dujella, A parametric family of elliptic curves, Acta Arith. 94 (2000), $87-101$

[6] A. Dujella And A. Pethő, Integer points on a family of elliptic curves, Publ. Math. Debrecen 56 (2000), 321-335.

[7] P. Erdős And J. L. Selfridge, The product of consecutive integers is never a power, Illinois J. Math. 19 (1975), 292-301.

[8] P. Erdős and R. L. Graham, On products of factorials, Bulletin of the Inst. of Math. Acad. Sinica 4 No. 2, (1976), 337-355.

[9] P. ERdös AND R. L. Graham, Old and new problems and results in combinatorial number theory, Monographie No. 28, L'Enseignement Math. Genéve (1980).

[10] A. Grelak and A. Grytczuk, On the diophantine equation $a x^{2}-b y^{2}=c$, Publ. Math. Debrecen 44 (1994), 191-199.

[11] F. Najman, Compact representation of quadratic integers and integer points on some elliptic curves, Rocky Mountain J. Math. 40 (2010), 1979-2002.

[12] The PARI-group, PARI/GP mathematics software (version 2.5.0), Bordeaux, 2011, http://pari.math.u-bordeaux.fr/.

[13] B. Rosser And L. Schoenfeld, Approximate formulas for some functions of prime numbers, Illinois J. Math. 6 (1962), 64-94.

[14] N. Saradha AND T. N. ShOREY, Squares in products of factorials, in preparation.

[15] M. Ulas, On products of disjoint blocks of consecutive integers, L'enseignement Math. 51 (2005), 331-334.

\section{Addresses of the authors :}

Department of Mathematics, University of Zagreb,

Bijenička cesta 30, 10000 Zagreb, Croatia

School of Mathematics, Tata Institute of Fundamental Research, Homi Bhabha Road, Mumbai - 400 005, India

Department of Mathematics, Indian Institute of Technology,

Powai, Mumbai - 400 076, India

\section{emails:}

duje@math.hr

fnajman@math.hr

saradha@math.tifr.res.in

shorey@math.iitb.ac.in 